\title{
Diagnosis and Management of Childhood Tuberculosis in Public Health Clinics in a Rural Area in the Philippines: Results from a Community Surveillance
}

\author{
Josephine G. Aldaba, ${ }^{1}$ Ma. Cecilia G. Ama, ${ }^{2}$ Aida M. Salonga, ${ }^{1}$ \\ Patrick G. Sylim, ${ }^{3}$ Jesus N. Sarol, Jr ${ }^{4}$ and Anna Lena Lopez ${ }^{1}$ \\ ${ }^{1}$ Institute of Child Health and Human Development, National Institutes of Health, University of the Philippines Manila, \\ ${ }^{2}$ Research Institute for Tropical Medicine \\ ${ }^{3}$ National TeleHealth Center, National Institutes of Health, University of the Philippines Manila, \\ ${ }^{4}$ National Teacher Training Center for the Health Professions, University of the Philippines Manila
}

\begin{abstract}
Background. Childhood tuberculosis (TB) remains a significant health problem worldwide despite the increase on its emphasis in national health programs.

Objective. This study aimed to describe how TB in children is identified and managed in a routine TB program in a rural setting in a high-burden country.

Methods. This is a prospective, community-based surveillance study in public rural clinics in the Philippines. Observations on case finding and management of TB in children as well as contact tracing in an existing TB program are described.

Results. Out of 266 children with presumptive TB, 41 (15.4\%) were cases of TB, 15 (5.6\%) had latent TB infection (LTBI), 81 (30.5\%) had TB exposure, and 129 (48.5\%) had no TB. There were 37 (90\%) TB cases who were clinically diagnosed. Ninety-three per cent (93\%) of children with TB disease were cured or completed treatment. Among 25 children targeted for isoniazid preventive therapy (IPT) for LTBI and TB exposure, only 12 (52\%) completed the recommended 6 months of IPT. Only 40 (43\%) children aged 0 to 4 years exposed to smear-positive TB cases were screened.
\end{abstract}

Conclusion. Barriers in the diagnosis, low IPT completion and problems in contact tracing may hinder successful implementation of TB programs for children.

Key Words: children, Mycobacterium tuberculosis, pediatric tuberculosis, Philippines

\section{INTRODUCTION}

Tuberculosis (TB) in the Philippines declined by more than half from 1990 to $2015^{1}$ and the country has mainly achieved this through the National Tuberculosis Control Program (NTP) with directly observed treatment, short-

Corresponding author: Josephine G. Aldaba, MD Institute of Child Health and Human Development Room 112, National Institutes of Health Building, University of the Philippines Manila 623 Pedro Gil Street, Manila, 1000 Philippines Telephone: +6322545205

Email: jga94@yahoo.com course (DOTS) as its centerpiece strategy. However, from 2003 to 2011, the proportion of children aged 0 to 14 years notified to the Philippines's NTP relative to other cases did not exceed $2 \%,{ }^{1}$ but with the change in case notification, the number of cases notified to the country's NTP increased in 2014 and 2015 to $12.1 \%^{2}$ and $9.6 \%$, respectively. ${ }^{3}$ 
Globally, it is estimated that there were 1 million new cases of childhood TB or $10 \%$ of the total incident cases, however, in 2015 children accounted for only $6.3 \%$ of the total cases. ${ }^{3}$ Although, there is increasing emphasis on childhood tuberculosis in national tuberculosis programs, previously, childhood tuberculosis has been neglected in the majority of national TB programs. ${ }^{4,5}$ This has been largely due to the difficulty in confirming childhood cases of TB, which presents with non-specific signs and symptoms that may elude diagnosis. ${ }^{6}$ The majority of childhood TB are paucibaciliary and hence are smear-negative, posing a dilemma for all programs, which focuses on acid-fast bacilli (AFB) smears as a screening tool., 2,6 Other widely used diagnostic tests, namely chest radiography and tuberculin skin testing, also have low yield. ${ }^{7}$ Current strategies have combined different clinical and diagnostic parameters to come up with a definitive diagnosis. More recently, newer diagnostic tests have been added to assist in confirming TB diagnosis in children. ${ }^{8}$

In the Philippines, a review of the NTP showed a wide variation in the proportion of smear-negative childhood TB relative to total smear-negative cases reported per region with some regions with no reported cases to some with more than $50 \%$ of the total. ${ }^{9}$

These variations may indicate that there may be different reasons that contribute to the neglect of childhood TB in health programs which are unique to a community or locality.

\section{MATERIALS AND METHODS}

\section{Setting and study procedures}

This is a prospective community-based surveillance study in San Juan, Batangas, using the existing NTP set-up, from May 14, 2013 to July 31,2015, as previously described. ${ }^{10}$ San Juan is a rural municipality in the Philippines with a population of 99,755 in 2013. It has a young population with children less than 15 years old comprising about $43 \%$ of the population. San Juan was chosen as the study site because of a cooperative local government which has control over the local health system and because its rural health unit conforms to the guidelines of the NTP as evidenced by its accreditation as a DOTS facility.

Briefly, the study used the NTP guidelines and case definitions for diagnosis of $\mathrm{TB}$, including those for children. ${ }^{11-13}$ Children in the NTP is defined as less than 15 years of age. Based on the NTP guidelines, passive surveillance included TB case finding among those symptomatic patients who presented themselves to the San Juan Rural Health Unit (RHU). Enhanced surveillance was conducted by the community health workers among household contacts of diagnosed and registered TB cases of the RHU as they were instructed by the NTP. The main diagnostic tool used was direct sputum smear microscopy (DSSM). For patients whose DSSM were negative, chest $\mathrm{x}$-ray (CXR) were requested. However, CXR is not provided for free in the NTP. In addition to the standard diagnostic tests offered in the NTP, sputum TB culture was added as part of the study.

For children who were presumptive TB cases but who could not provide sputum samples for DSSM, CXR and tuberculin skin testing (TST) were performed following NTP guidelines. TST was also done during the evaluation of children 0-4 years old who were household contacts of index cases when they presented at the local health center. For children aged 0-4 years who were household contacts of an index case whose TST was negative, TST was repeated after three months.

Information (age, sex, source of patient whether public or referred by private physician, presenting signs and symptoms, history and nature of TB exposure, number and age group of household members, laboratory test results DSSM, TB Culture, CXR, diagnosis, TB classification, and outcome) was collected on paper and entered to Samsung Galaxy chat phones via an Android-based application (mTB app) specifically programmed for the study. The information was then sent through short messaging service (SMS) to a database through a gateway. Children were diagnosed as either TB disease, latent TB infection, TB exposure, or no TB. Those with TB disease were classified as either pulmonary or extrapulmonary $\mathrm{TB}$ and as bacteriologicallyconfirmed TB or clinically-diagnosed TB. Possible outcomes of TB patients were recorded as: 1) cured (a patient whose sputum became negative by smear or culture in the last month of treatment and on at least one previous occasion), 2) treatment completed, 3) treatment failed, 4) died, 5) lost to follow up (one whose treatment was interrupted for two consecutive months or more), and 6) not evaluated (patients who did not complete diagnostic procedures so subsequently was not diagnosed). To be diagnosed as having TB disease, children aged 0-14 years old must fulfill 3 out of the following 5 criteria: 1 ) TB symptomatic which is the presence of at least 3 of the 6 signs and symptoms suggestive of $\mathrm{TB}$ (coughing or wheezing for $\geq 2$ weeks, especially if unexplained; unexplained fever of $>2$ weeks, after common causes such as malaria and pneumonia have been excluded; loss of weight/failure to gain weight/weight faltering/loss of appetite; failure to respond to 2 weeks of appropriate antibiotic therapy for lower respiratory tract infection; failure to regain previous state of health after viral infection of exanthem (e.g. measles); and fatigue/reduced playfulness/lethargy; 2) exposure to an active TB case (adult or adolescent); 3) positive tuberculin skin testing; 4) CXR suggestive of $\mathrm{TB}$; and 5 ) other diagnostic procedures. A child who had no signs and symptoms presumptive of TB nor radiologic or laboratory evidence, but tested positive on tuberculin skin testing was classified as having latent TB infection (LTBI). A child who was in close contact with an active adult TB case, but without any signs and symptoms of TB, with negative TST reaction, and no radiologic or laboratory findings suggestive of TB was classified as TB 
exposure. A child who did not fall under any of the previously described diagnoses or who was assessed by the physician as having another disease was considered as having no $\mathrm{TB}$, for the purposes of this study.

As an accredited DOTS provider, all NTP health staff members in the RHU have undergone standard NTP training. The Municipal Health Officer (MHO), rural health physicians (RHP), and the NTP Coordinator were certified to have undergone training on case finding and management of tuberculosis in children.

Treatment of diagnosed TB cases was provided as part of NTP guidelines. Children aged $<5$ years who were assessed to have LTBI were placed on isoniazid preventive therapy (IPT). Asymptomatic children aged $<5$ years who were exposed to bacteriologically confirmed source cases with negative TST but had no CXR done may be started on IPT.

\section{Data analysis}

Information obtained from the $\mathrm{TB}$ surveillance was reviewed. Data were age disaggregated into the following age groups: 0 to less than 5 years old, 5 to 9 years old, 10 to 14 years old, and 15 years and older, tabulated and analyzed using Microsoft Excel for Mac version5.

Descriptive statistics were calculated. Information on the number of cases was assessed at regular intervals of the surveillance to ensure that cases were captured and compared with baseline data submitted to the NTP in the preceding 16 months prior to surveillance. The number and age groups of the household contacts of those who were diagnosed with TB were also retrieved. For children who were enrolled, we reviewed their patterns of exposure. Demographic variables were tabulated among bacteriologically and clinically confirmed pediatric TB patients.

\section{Ethics}

This study underwent ethical review and was approved by the University of the Philippines Manila-Research Ethics Board (UPMREB-2012-0355). Written informed consent was provided by all adults and all parents/guardians of children from birth to $<18$ years old. Assent was obtained from all children aged 7-17 years. The study was anchored on the public health program of NTP thus all TB work-up, including the TB culture, of enrolled subjects went through the RHU DOTS staff and were relayed personally to the subjects. The study staff only had access to results of patients who gave consent.

\section{RESULTS}

From 14 May 2013 to 31 July 2015, there were 318 children (presumptive TB and exposure) who were enrolled in the study (Table 1). Children represented $19.6 \%$ of all 1,622 presumptive TB cases. Among these 318 children, 266 children completed the follow-up for evaluation (Table 1). Out of the 266, $41(15.4 \%)$ were diagnosed to have TB
Table 1. Characteristics of children who were screened for TB and those with complete information

\begin{tabular}{lll} 
& $\begin{array}{c}\text { All children } \\
\text { screened for TB } \\
\mathbf{N = 3 1 8} \\
\mathbf{n}(\%)\end{array}$ & $\begin{array}{c}\text { Children who } \\
\text { completed screening } \\
\mathbf{N}=\mathbf{2 6 6} \\
\mathbf{n}(\%)\end{array}$ \\
\hline Age group & & \\
0- 4 years old & $141(44)$ & $129(48)$ \\
5- 9 years old & $127(40)$ & $113(42)$ \\
10- 14 years & $50(16)$ & $24(9)$ \\
\hline Male & $172(54)$ & $143(54)$ \\
\hline Exposure & $153(48)$ & $111(42)$ \\
Within household & $137(43 \%)$ & $102(38)$ \\
\hline Referral source & & \\
Public & $222(70)$ & $181(68)$ \\
Private & $70(22)$ & $65(24)$ \\
Not recorded & $26(8)$ & $20(8)$ \\
\hline
\end{tabular}

disease, 15 (5.6\%) had LTBI, 81 (30.5\%) had TB exposure, and $129(48.5 \%)$ had no TB.

Children diagnosed with TB disease comprised only 9 $\%$ of the total 468 adults and children diagnosed with TB during the study period. Among children with TB disease, the average age was 5.7 years (median 5 years, range 11 months to 14 years), however, bacteriologically-confirmed cases were older. The majority $(n=37,90.2 \%)$ of TB disease cases were clinically confirmed (Table 2).

\section{Bacteriologically-confirmed childhood TB}

Out of 266 children with complete information, 43 (16\%) were able to provide a sputum sample for DSSM. The youngest child who was able to provide a sample was 6 years old. Four (9.3\%) children were positive by culture, none showed resistance. Three children, who were positive by both culture and smear, were aged 14 years, and presented with cough and fever of more than 2 weeks, weight loss and fatigue. Two of these children had known household exposure to TB. One, a 10-year-old, had a negative smear, but positive culture and presented with only two symptoms: weight loss and fatigue; and was exposed to a parent who was diagnosed with TB (Table 2).

\section{Use of clinical algorithm in diagnosis of childhood TB}

Out of 266 children, 71 (27\%) fulfilled the criteria of having 3 of the 6 symptoms identified with TB and were assessed as TB symptomatic. A total of 195 (73\%) were either asymptomatic or had less than 3 symptoms. The most common symptom was cough of more than 2 weeks which was reported in 165 (62\%) children.

Among those diagnosed with $\mathrm{TB}$ disease $(\mathrm{N}=41)$, 19 (46\%) were TB symptomatic while 22 (54\%) had 2 or less clinical symptoms. Among those who were not considered as TB symptomatic was a 10-year-old who had positive sputum culture. The most common symptoms in all TB disease cases were: cough $(\mathrm{n}=32,78 \%)$, loss of appetite/weight loss $(n=25,61 \%)$ and fever $(n=16,39 \%)$. 
Table 2. Characteristics of patients with bacteriologically-confirmed TB and clinically-diagnosed TB

\begin{tabular}{|c|c|c|c|}
\hline & $\begin{array}{l}\text { Bacteriologically- } \\
\text { confirmed TB disease } \\
n=4 \\
n(\%)\end{array}$ & $\begin{array}{l}\text { Clinically-diagnosed } \\
\text { TB disease } \\
\mathbf{n = 3 7} \\
\mathbf{n}(\%)\end{array}$ & $\begin{array}{c}\text { Total with } \\
\text { TB disease } \\
\mathrm{n}=41 \\
\mathrm{n}(\%)\end{array}$ \\
\hline \multicolumn{4}{|l|}{ Age Group } \\
\hline 0 to 4 & $0(0)$ & $20(54)$ & $20(49)$ \\
\hline 5 to 9 & $0(0)$ & $15(41)$ & $15(36)$ \\
\hline 10 to 14 & $4(100)$ & $2(5)$ & $6(15)$ \\
\hline Male & $2(50)$ & $21(56.8)$ & $23(56.1)$ \\
\hline Presence of BCG scar & $2(50)$ & $28(75.7)$ & $30(73.2)$ \\
\hline Known TB exposure & $3(75)$ & $10(27)$ & $13(31.7)$ \\
\hline Tuberculin skin test positive $^{a}$ & $0(0)$ & $19(51.3)$ & $19(46)$ \\
\hline Chest $\mathrm{x}$-ray compatible with $\mathrm{TB}^{\mathrm{b}}$ & $2(50)$ & $25(67.5)$ & $27(66)$ \\
\hline Fulfilled 3 of the 5 criteria to be considered as TB symptomatic & $3(75)$ & $16(43.2)$ & $19(46)$ \\
\hline \multicolumn{4}{|l|}{ Clinical presentation } \\
\hline Cough 2 weeks or more & $3(75)$ & $26(70.3)$ & $29(70.7)$ \\
\hline Loss of appetite/weight faltering & $4(100)$ & $21(56.8)$ & 25 (60.9) \\
\hline Fever 2 weeks or more & $3(75)$ & $14(37.8)$ & $17(41.5)$ \\
\hline Fatigue/Lethargy & $3(75)$ & $12(32.4)$ & $15(36.6)$ \\
\hline Failure to respond to antibiotic therapy & $0(0)$ & $6(16.2)$ & $6(14.6)$ \\
\hline Lymphadenopathy & $0(0)$ & $5(13.5)$ & $5(12.1)$ \\
\hline Failure to regain previous state of health & $0(0)$ & $2(5.4)$ & $2(48.8)$ \\
\hline \multicolumn{4}{|c|}{$\begin{array}{l}\text { Among bacteriologically confirmed cases, no TST were done. Among clinically-diagnosed, twenty-six (26) had TST and came back for reading of } \\
\text { results. Of this number, } 19 \text { were TST positive. Eleven (11) of the } 37 \text { clinically-diagnosed cases did not have TST or results were unknown. } \\
\text { Among the } 4 \text { bacteriologically confirmed, } 2 \text { had CXRs that were read as positive for TB; } 2 \text { were not done. Among clinically-diagnosed, } 25 \text { had CXRs } \\
\text { that were read as positive, } 12 \text { had no CXRs done or unknown results. }\end{array}$} \\
\hline
\end{tabular}

Furthermore, among these 41 children diagnosed with TB disease, 13 (32\%) had known TB exposure among which, 11 occurred within the household, and the majority $(n=10)$ of the identified source was one or both parents, and 8 of the source cases were smear positive.

CXRs were not provided for free in the NTP and only 27 (10\%) had CXR. All 27 children had CXR findings suggestive of TB and all were diagnosed with TB disease.

Of the 266 children, 232 (87\%) had TST, among which $34(13 \%)$ were positive, 158 (59\%) were negative and 40 (15\%) did not return for the reading of the skin test. The latter were referred by private physicians. Of the 41 diagnosed with TB disease,19 (46\%) had positive TST, 7 (17\%) had negative results while 15 (37\%) children had no TST.

Only 111 (42\%) had exposure to a known TB case, among which 102 (92\% of those with exposure) occurred within the household and 78 (70\%) of these household exposures were to sputum smear positive source cases. Of the 78 children household contacts of smear positive source cases, 42 (54\%) were asymptomatic and were classified as either TB exposure $(n=34,44 \%)$ or latent TB infection $(n=6,8 \%)$. Of the 36 (46\%) symptomatic children who were household contacts of smear positive source cases, 4 (5\%) were diagnosed with TB disease, 25 (32\%) had TB exposure, 6 (8\%) had LTBI and 1 (1\%) had no TB.

\section{Outcomes}

Of the 41 children diagnosed with TB disease, 38 (93\%) children were enrolled in the NTP and started on anti-TB treatment. All were new cases of TB and all had pulmonary
TB; 35 children completed treatment and 3 children were cured. For the 3 children who were not enrolled in the NTP, one moved residence and was lost to follow up, while 2 opted to have their treatment with private physicians. There were no deaths among the children diagnosed with TB disease.

Among the 15 children diagnosed with LTBI, 8 were younger than 5 years and were recommended to be started on Isoniazid Preventive Therapy (IPT) according to NTP guidelines. However, only 2 (25\%) were started on IPT. One was lost to follow-up and the other completed therapy.

Of the 81 children classified as TB exposed, 56 were less than 5 years old and 48 were exposed to smear positive patients, and thus were recommended to be started on IPT. Of the 48, only 21 (44\%) were started on IPT. Among the 21 started on IPT, only 11 completed therapy; 1 transferred residence and 9 were lost to follow-up.

Overall, of the 23 children with LTBI and TB exposure who were started on IPT, only $12(52 \%)$ completed the recommended 6 months of IPT. Feedback from health workers in the study identified the following causes for the low compliance: parents may not have understood why asymptomatic children had to take medications for a prolonged period, and the inconsistent availability of isoniazid in the public health facility.

\section{Contact tracing}

Among the 468 cases of $\mathrm{TB}$ in adults and children included in the surveillance, household contacts of both bacteriologically and clinically-diagnosed TB cases (Table 3) were followed. No contact information was available from 
Table 3. Number of household contacts of 442 adults and children with diagnosed TB enrolled during the surveillance

\begin{tabular}{|c|c|c|c|}
\hline Age group & $\begin{array}{c}\text { No. of contacts of } 256 \\
\text { bacteriologically-confirmed } \\
\text { TB patients } \\
\mathrm{n} \text { (\% of total) }\end{array}$ & $\begin{array}{l}\text { No. of contacts of } 154 \\
\text { clinically-diagnosed adult } \\
\text { and adolescent TB patients } \\
\text { n (\% of total) }\end{array}$ & $\begin{array}{c}\text { No. of contacts of } 32 \\
\text { clinically-diagnosed } \\
\text { pediatric patients } \\
n \text { (\% of total) }\end{array}$ \\
\hline $0-4$ years old & $112(11.8)$ & $50(9.5)$ & $2(2.0)$ \\
\hline 5- 9 years old & $122(12.9)$ & $71(13.5)$ & $15(14.8)$ \\
\hline 10- 14 years old & 145 (15.3) & $84(16.0)$ & $11(10.9)$ \\
\hline 15 years old and older (adults) & $566(60.0)$ & $321(61.0)$ & $73(72.3)$ \\
\hline Total & 945 & 526 & 101 \\
\hline
\end{tabular}

a Out of 274 bacteriologically confirmed TB cases there were: 227 DSSM positive, 45 culture positive but DSSM negative, and 2 positive but DSSM and culture negative patients. Eighteen (18) cases did not have any recorded household contacts.

$b$ Adolescent subjects are those who are 10 to 14 years old. Adults are those who are 15 years old and above.

18 bacteriologically confirmed and 8 clinically confirmed cases. The 442 patients with household contact information had a total of 1,572 household contacts, or 3.6 contacts per index case.

Among 112 bacteriologically confirmed TB cases, 92 were smear positive. When source case information from children aged 0 to 4 years in the study were cross-checked with the names of the 92 patients with smear positive results, only $40(43 \%)$ household contacts in this age group were screened for TB.

\section{Recording childhood TB cases and feedback}

Information at 3 months into the surveillance (14 May 2013 to August 2013) on childhood TB was compared with baseline information from January 2011 to April 2013, prior to surveillance. At 3 months of surveillance, no case of childhood TB was reported, which was similar to the preceding 16 months prior to surveillance. Staff complained of the lack of anti-TB medications for children in the RHU. Because there were no medicines, staff did not detect cases in children and instead referred them to private physicians for diagnosis and management. Discussions with the RHU staff further revealed other issues: absence of agedisaggregated reporting so monitoring of children with TB was inconsistent, lack of training on TB in children by the frontline staff, inconsistent implementation of contact tracing so exposed children were not screened, transportation costs and problems with diagnostics such as no purified protein derivative (PPD) for TST, no follow-up for TST readings, CXR not being performed and young children being unable to provide sputum.

Following age disaggregation of cases, augmentation of anti-TB medications for children and re-training of frontline staff, an increase in the number of cases were seen by the $6^{\text {th }}$ month of the surveillance.

\section{DISCUSSION}

We present the experience on the diagnosis and management of childhood TB in public health clinics in a rural area in the Philippines. We identified 41 cases of childhood TB in a span of 26.5 months or $9 \%$ of all notified TB cases in San Juan, lower than the estimated 15\% to $18 \%$ of the total TB cases in the country. ${ }^{14}$ Only 4 of the diagnosed childhood TB cases were bacteriologically confirmed, the rest were diagnosed clinically. Among diagnosed TB cases the majority (93\%) completed treatment or were cured, however almost half (48\%) did not complete their IPT course. This is a missed opportunity in TB control since isoniazid reduces the risk of progression to disease by $59 \%$ in children $<15$ years of age. ${ }^{15}$ We believe that we were able to capture most of the household contacts considering that the average household size in San Juan in 2010 was 4.4. ${ }^{16}$ However, only $43 \%$ of children aged 0 to 4 years who were household contacts of smear positive TB cases were screened. Our results on contact screening were somewhat lower compared to a cross-sectional study conducted in urban settings in the Philippines, ${ }^{17}$ wherein $57.3 \%$ of listed household contacts of diagnosed TB cases who were aged 0 to 4 years were screened for TB in public health facilities run by local governments. Due to the devolved nature of the health care system in the Philippines, implementation of health programs may be inconsistent nationwide, particularly in rural areas. Although the WHO recommends tracing and provision of IPT for all children $<5$ years old who are household contacts of sputum smear-positive cases once TB disease has been ruled out, ${ }^{18}$ contact tracing is inconsistently implemented. ${ }^{19} \mathrm{~A}$ health needs assessment framework was used to address this policy gap $^{19}$ in Indonesia which showed that only $8 \%$ of eligible child contacts returned to the clinic for screening. ${ }^{20}$

The initial low or absent cases of childhood TB identified in the first few months of our surveillance in 2013, was probably similar to the experience of some other areas in high burden countries like the Philippines. Some of the problems identified in our study during discussions with health care workers that affected childhood TB identification and inconsistent implementation of contact tracing such as stockouts of anti-TB drugs for children, lack of diagnostics such as PPD and transportation costs were also mentioned as possible reasons in other studies. ${ }^{17,21}$

Our study had several limitations. First, we only included ambulatory cases diagnosed in the public health clinics in San Juan. We missed the more severe cases, including deaths due to childhood TB. These cases would likely have been sent to hospitals with subspecialty care that are only available outside the municipality of San Juan. Second, we also 
missed ambulatory cases that have been brought to private physicians; $15 \%$ of children with TST in our study did not return for reading and most of these children returned to their private physicians for reading. In previous studies among TB patients, about half preferred to go to private physicians for initial care. ${ }^{22,23}$ Third, only $10 \%$ of children had CXRs done and it is likely that those children who were considered as TB exposure or LTBI may have CXR findings suggestive of $\mathrm{TB}$, thus necessitating treatment. Fourth, we only identified 15 cases of LTBI, substantially lower than expected. It is estimated that the Philippines has the $6^{\text {th }}$ highest burden of LTBI cases with 3.37 million LTBI cases in children $<15$ years old. ${ }^{24}$ This may be due to problems with contact tracing which was not consistently performed by community health workers who were also working on other programs in the community. Furthermore, travel costs of household contacts, including those who were asymptomatic had to be paid by the families themselves deterring visits to the health center for assessment.

Our study confirms the barriers in the diagnosis and management of TB in children. Depending on signs and symptoms that are not specific to TB together with inconsistent availability of CXR hinders accurate diagnosis. In the joint review of the Philippines' NTP, health workers complained of complicated algorithms for childhood TB. ${ }^{9}$ Simplified algorithms for identification of TB in children and its inclusion with the adult case identification and emphasis on contact tracing were introduced in the Revised Manual of Procedures of the NTP in 2014. Close monitoring of compliance in contact tracing implementation should be performed. If children can expectorate, sputum should be collected for bacteriogical confirmation. Use of more sensitive tests such as Xpert MTB/RIF particularly in children with paucibaciliary disease will increase the yield and hence likely improve case detection in children.

Childhood TB represents recent transmission of tuberculosis and should therefore be used as a sentinel event that requires improved TB control. We identified several constraints in the program; addressing these constraints will contribute to the identification, compliance to treatment and improve outcomes for childhood TB particularly at the community level.

\section{Acknowledgments}

The authors would like to thank the local government, rural health units, and the people of San Juan, Batangas, without whose participation and support, this study could not have been possible. This study was funded by the Hanako Foundation. Hanako Foundation had no role in the design, implementation, collection, analysis, and interpretation of data; in the writing of the manuscript; and in the decision to submit the manuscript for publication.

\section{Statement of Authorship}

All authors have approved the final version submitted.

\section{Author Disclosure}

All authors have declared no conflict of interest.

\section{Funding Source}

This research was funded by the Hanako Foundation.

\section{REFERENCES}

1. Vianzon R, Garfin AM, Lagos A, Belen R. The tuberculosis profile of the Philippines, 2003-2011: advancing DOTS and beyond. Western Pac Surveill Response J.2013;4(2):11-16. doi: 10.5365/ WPSAR.2012.3.4.022.

2. World Health Organization. Global Tuberculosis Report 2015 [Online]. 2015 [cited November 2017]. Available from http://www. who.int/tb/publications/global_report/gtbr15_main_text.pdf.

3. World Health Organization. Global Tuberculosis Report 2016 [Online]. 2016 [cited 2017 November]. Available from http://www. who.int/tb/publications/global_report/gtbr2016_executive_summary. pdf.

4. Donald PR, Maher D, Qazi S. A research agenda to promote the management of childhood tuberculosis within national tuberculosis programmes. Int J Tuberc Lung Dis. 2007;11(4):370-80.

5. Detjen A, Gale M, Baena IG, et al. Roadmap for childhood tuberculosis: Towards zero deaths. [Online]. World Health Organization; 2013. [cited 2017 November]. Available from: http://apps.who.int/iris/ bitstream/10665/89506/1/9789241506137_eng.pdf.

6. Eamranond P, Jaramillo E. Tuberculosis in children: reassessing the need for improved diagnosis in global control strategies. Int J Tuberc Lung Dis. 2001;5(7):594-603.

7. Batra S, Ayaz A, MurtazaA, Ahmad S, Hasan R, Pfau R. Childhood tuberculosis in household contacts of newly diagnosed TB patients. PLoS One. 2012;7(7):e40880. doi: 10.1371/journal.pone.0040880. Epub 2012 Jul 31.

8. World Health Organization. Guidance for national tuberculosis programmes on the management of tuberculosis in children [Online]. 2014 [cited 2016 May]. Available from: http://apps.who.int/ medicinedocs/documents/s21535en/s21535en.pdf.

9. Hanson C. Joint Tuberculosis Program Review Philippines 2013. Presented at the Joint Technical Program Review of the National Tuberculosis Program in the Philippines, 25 August - 6 September 2013. Manila.

10. Lopez AL, Aldaba JG, Ama CG, et al. Surveillance for tuberculosis in a rural community in the Philippines. Int J Tuberc Lung Dis. 2016;20(11):1495-1500. doi: 10.5588/ijtld.16.0027.

11. Philippine Department of Health. National Tuberculosis and Control Program: Manual of Procedures. Manila: Department of Health; 2005.

12. Philippine Department of Health Administrative Order 2008-011. Revised Guidelines for Implementing Tuberculosis Control Program in Children, Manila; 2008.

13. Philippine Department of Health. National TB Control Program: Manual of Procedures. 5th edition. Manila: Department of Health; 2014. p178.

14. Dodd PJ, Gardiner E, Coghlan R, Seddon JA. Burden of childhood tuberculosis in 22 high-burden countries: a mathematical modelling study. Lancet Glob Health [Online]. 2014 [cited 2017 November];2(8):e453-e459. Available from: http://www.thelancet. com/pdfs/journals/langlo/PIIS2214-109X(14)70245-1.pdf .

15. Ayieko J, Abuogi L, Simchowitz B, Bukusi EA, Smith AH, Reingold A. Efficacy of isoniazid prophylactic therapy in prevention of tuberculosis in children: a meta-analysis. BMC Infect Dis. 2014;14:91. doi: 10.1186/1471-2334-14-91. PubMed PMID: 24555539.

16. Philippine Statistics Authority. Philippines - Census of Population and Housing 2010 [Online]. 2010 [cited 2017 July]. Available from: http:// psa.gov.ph/psada/index.php/catalog/64/datafile/F9/V147.

17. Coprada L, Yoshimatsu S, Querri A, et al. A review of tuberculosis contact investigations in the poor urban areas of Manila, the Philippines. Public Health Action. 2016;6(4):220-25. doi: 10.5588/pha.16.0042. 
18. World Health Organization. Guidance for National Tuberculosis Programmes on the Management of Tuberculosis in Children. Geneva: World Health Organization; 2014. Chapter 5 Prevention of TB in children; p. 43-54.

19. Hill PC, Rutherford ME, Audas R, van Crevel R, Graham SM. Closing the policy- practice gap in the management of child contacts of tuberculosis cases in developing countries. PLoS Med. 2011;8(10):e1001105. doi: 10.1371/journal.pmed.1001105.

20. Rutherford ME, Ruslami R, Anselmo M, et al. Management of children exposed to Mycobacterium tuberculosis: a public health evaluation in West Java, Indonesia. Bull of the World Health Organ [Online]. 2013;91(12):932-41a. [cited 2017 July] doi: 10.2471/blt.13.118414.

21. Weaver MS, Lonnroth K, Howard SC, Roter DL, Lam CG. Interventions to improve adherence to treatment for paediatric tuberculosis in low- and middle-income countries: a systematic review and meta-analysis. Bull of the World Health Organ [Online]. 2015;93(10):700-11B. [cited 2017 July]; doi: 10.2471/BLT.14.147231. Epub 2015 Jun 23.
22. Auer C, Sarol J Jr., Tanner M, Weiss M. Health seeking and perceived causes of tuberculosis among patients in Manila, Philippines. Trop Med Int Health. 2000;5(9):648-56.

23. Tupasi TE, Radhakrishna S, Co VM et al. Bacillary disease and health seeking behavior among Filipinos with symptoms of tuberculosis: implications for control. Int J Tuberc Lung Dis. 2000;4(12):1126-32.

24. Houben RM, Dodd PJ. The global burden of latent tuberculosis infection: a re-estimation using mathematical modelling. PLoS Med. 2016 Oct 25;13(10):e1002152. doi: 10.1371/journal.pmed.1002152. eCollection 2016 Oct.

\title{
Have you read the current trends in
}

Medical and Health Research in the Philippines?

\section{Acta Medica Philippina} The National Health Science Journal

\author{
Access Online: www.actamedicaphilippina.org
}

